\title{
HOMENAJE A VICKI CASSMAN, PH.D. (2 DE MARZO DE 1957 - 6 DE AGOSTO DE 2020)
}

\author{
Recordando a Vicki
}

\author{
Bernardo Arriaza ${ }^{1}$
}

Informamos a la comunidad la triste noticia del fallecimiento de Vicki Cassman, después de una larga lucha contra el cáncer. Vicki fue una experta en conservación de materiales arqueológicos y colaboró desinteresadamente con varios museos chilenos desde 1983. Primero fue invitada por la Universidad de Tarapacá y posteriormente auspiciada por la Comisión Fulbright, para contribuir a mejorar el manejo de colecciones y las preservación de textiles arqueológicos del Museo San Miguel de Azapa (Universidad de Tarapacá, Arica).

Vicki obtuvo su licenciatura en historia del arte en la Universidad de California, Davis. Luego dos maestrías: una en ciencias textiles y otra en conservación de arte (Winterthur, Delaware). Posteriormente, en 1997 obtuvo su doctorado en arqueología en Arizona State University. Su tesis doctoral se centró en la temática de etnicidad y textiles arqueológicos de Arica: "Reconsideration of Prehistoric Ethnicity and Status in Northern Chile: The Textile Evidence" (Department of Anthropology, Arizona State University, Tempe. Catalog No.9809447,

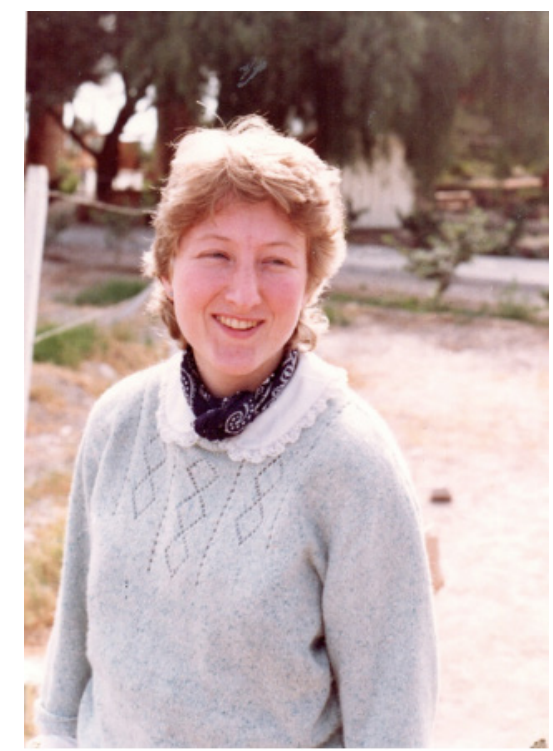

Dra. Vicki Cassman, Museo San Miguel de Azapa, Arica, 1984.
University Microfilms, Ann Arbor). Después de obtener su doctorado fue profesora de la Universidad de Nevada, Las Vegas (Departamento de Antropología, 1999-2006) y en la Universidad de Delaware, Newark, Delaware (Departamento de Conservación de Arte, 2006 en adelante). En ambas Universidades norteamericanas y en Chile, contribuyó a la formación de muchos profesionales que hoy la recuerdan con mucho cariño. Además, participó en importantes proyectos de conservación como, por ejemplo, en los restos del hombre de Kennewick, Washington State (Estados Unidos) de 8.500 años de antigüedad y en la conservación para exhibición y traslado de los famosos restos de Lucy, un homínido de Etiopia de cerca de 3 millones de años de antigüedad.

Vicki fue una docente por excelencia, extremadamente dedicada y amable. Por ello recibió númerosos honores, incluyendo becas Fullbright, y fue distinguida por sus labores de excelencia en la docencia con los premios "Outstanding Service Award" y "Excellence in Advising and Mentoring Award" del College of Arts and Sciences de la Universidad de Delaware. Además de su calidad como docente e investigadora, y de su simpatía a flor de piel, dominaba varios idiomas, como el sueco y el español. Su contribución fue amplia tanto en el ámbito de la conservación como de la arqueología. Podemos por ejemplo, señalar su libro Human Remains: Guide for Museums and Academic Institutions (AltaMira Press, Rowman \& Littlefield Publishers, Inc. Lanham, Maryland. 2007, junto a N. Odegaard y J. Powell), y múltiples contribuciones en la revista Chungara, donde además era parte del comité asesor editorial.

Siguiendo sus deseos sus cenizas fueron depositadas en el Bosque Better Place en las montañas de Santa Cruz, California, Estados Unidos. A Vicki la sobreviven su hijo Victor Arriaza, su hermano Peter Cassman, muchos tíos y tías, primos, sobrinas y sobrinos, que residen en Estados Unidos, Suecia y Chile. En su memoria, Vicki se sentiría muy feliz si donaras a una organización de lucha contra el cambio climático o a una organización benéfica de atención médica.

Quiero cerrar estas palabras de despedida, recordando que fuiste una muy linda persona, colega y compañera.

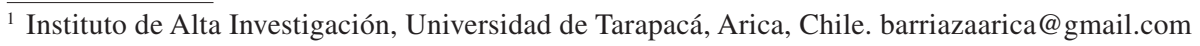


Ciertamente extrañaremos tu simpatía y amistad, junto a los diálogos permanentes sobre la ética, el manejo de colecciones y la conservación preventiva. Fuiste una fuente de inspiración para mí y para tus estudiantes. Descansa en paz compañera, tu siembra intelectual no fue en vano y la brisa de los bosques donde ahora descansas nos dará nuevos bríos para continuar con los desafíos del trabajo de conservación del patrimonio cultural.

\section{Resumen de algunas de sus publicaciones científicas}

Arriaza, B. y V. Cassman 1988. ¿Se está produciendo un arqueocidio? Chungara Revista de Antropología Chilena 20:69-73.

Arriaza, B. y V. Cassman 1997. Mummies in South America. En Encyclopedia of the History of Science, Technology, and Medicine in Non Western Cultures, editado por H. Selaine, pp. 750-752. Springer, Dordrecht.

Arriaza, B., V. Standen, V. Cassman y C. Santoro 2008. Chinchorro, the pioneers of the Atacama coast. En Handbook of South American Archaeology, editado por H. Silverman y W.H. Isbell, pp. 45-58. Springer, New York.

Arriaza, B., V. Standen, J. Heukelbach, V. Cassman y F. Olivares 2014. Head combs for delousing in ancient Arican populations: scratching for the evidence. Chungara Revista de Antropología Chilena 46 (4):1-11.

Belmonte, E., M. Ortega, P. Arévalo, V. Cassman y L. Cartmell 2001. Presencia de la hoja de coca en el ajuar funerario de tres cementerios del periodo Tiwanaku: AZ 140, AZ.6 y PLM.3. Chungara Revista de Antropología Chilena 31 (1):125-136

Blatt, S., B. Redmond, V. Cassman y P. Sciulli 2010. Dirty teeth and ancient trade: evidence of cotton fibers in human dental calculus from Late Woodland, Ohio. International Journal of Osteoarchaeology 21:669-678.

Cassman, V. 1989. Simbiosis entre el museo y la arqueología. Chungara Revista de Antropología Chilena 23:93-109.

Cassman, V. 1992. Organización del trabajo de conservación. En Encuentro Regional de Expertos Sobre Conservación de Textiles Precolombinos. Informe Final, pp. 197-201. PNUD-UNESCO/Getty Conservation Institute Conference Proceedings Publication.

Cassman, V. 1992. El catastro: primer paso en la conservación preventiva. En Encuentro Regional de Expertos Sobre Conservación de Textiles Precolombinos. Informe Final, pp. 189-196. PNUD-UNESCO/Getty Conservation Institute Conference Proceedings Publication.

Cassman, V. 2000. Prehistoric ethnicity and status based on textile evidence from Arica, Chile. Chungara Revista de Antropología Chilena 32(2):253-257.

Cassman, V. 2000. Prehistoric Andean ethnicity and status: the textile evidence. En Beyond Cloth and Cordage: Archaeological Textiles Research in the Americas, editado por P. Drooker y L. Webster, pp. 253-266. University of Utah Press, Salt Lake City.

Cassman, V. 2001. Artifacts associated with human remains. En Human Remains: Conservation, Retrieval, Analysis, editado por E. Williams, pp. 57-59. Archaeopress (BAR International Series 934), Oxford.

Cassman, V., L. Cartmell y E. Belmonte 2003. Coca as symbol and labor enhancer in the Andes: a historical overview. En Stimulating Trade: Drugs, Labor and European Expansion, editado por W. Jankowiak y D. Bradburd, pp. 149-158. University of Arizona Press, Tucson.

Cassman, V. y N. Odegaard 2004. Human remains and the conservator's role. Studies in Conservation 49 (4):271-282.

Cassman, V., R. Layton, P.G. Stone y J. Thomas 2004. Destruction and conservation of cultural property. American Antiquity 69 (1):162-163 .

Needles, H.L., V. Cassman y M. Collins 1986. Mordanted natural dyed wool and silk fabrics: light and burialinduced changes in the color and tensile properties of mordanted natural dyed silk and wool textiles. En Historic Textiles and Paper Materials: Conservation and Characterization, editado por H.L. Needles y H. Zeronian, pp. 199-210. American Chemical Society, Washington, D.C.

Oakland, A. y V. Cassman 1995. Andean tapestry: structure informs the surface. Art Journal 54 (2):33-39.

Odegaard, N. y V. Cassman 2014. Authentication and conservation in archaeological science. En Encyclopedia of Global Archaeology, editado por C. Smith, pp. 702-711. Springer, New York

Odegaard, N. y V. Cassman 2016. The conservation of human remains: ethical questions and experiences in America. Technè 44:18-21.

Odegaard, N. y V. Cassman 2019. Storage of human remains. En Preventive Conservation: Collection Storage, editado por L. Elkin y C.A. Norris, pp. 100-120. Society for the Preservation of Natural History Collections, New York.

Trimble, M., N. Odegaard, V. Cassman y M. Teresa 2001. Curation of the Kennewick remains. En Human Remains: Conservation, Retrieval, Analysis, editado por E. Williams, pp. 237-248. Archaeopress (BAR International Series 934), Oxford. 